\title{
Working heart model of heterotopic heart-lung transplantation in rats
}

\begin{abstract}
A technique for a working heart model of heterotopic heart-lung transplantation is described. The donor aorta and left pulmonary artery are anastomosed to the recipient's abdominal aorta in an end-to-side fashion. Arterial blood from the recipient's abdominal aorta enters the donor left heart via the donor left lung and is ejected into the donor ascending aorta by the transplanted left ventricle. With this model, the donor left ventricle performs work comparable to that of the native heart without hypoxia. This model is easily reproducible and can be applied to various studies, including long-term assessments of cardiac remodeling, long-term efficacy of cardiac preservation, and posttransplantation ventricular performance under more physiologic conditions. (J THORAC CARDIovasc SuRG 1994; 107:210-5)
\end{abstract}

Toshiyuki Maruyama, MD, Marc T. Swartz, Lawrence R. McBride, MD, and

D. Glenn Pennington, MD, St. Louis, Mo.

$\mathrm{H}$ eterotopic cardiac transplantation in small animals is a useful, economical method and has been widely used in studying many areas, including heart transplantation, ${ }^{1}$ cardiac preservation, ${ }^{2}$ and cardiac remodeling. ${ }^{3-5}$ However, in most of the techniques described, the transplanted heart is in a virtually nonworking state. The lack of myocardial work leads to cardiac atrophy, ${ }^{6,7}$ which has limited further application of this model in areas such as chronic assessments of myocardial preservation techniques and more physiologic approaches to studies related to transplantation.

Recently, we developed a long-term working model of heterotopic heart-lung transplantation in rats to study cardiac remodeling. The purpose of this article is to describe the technique and to provide preliminary hemodynamics obtained while using this model.

\section{Materials and methods}

The schema of blood flow in the completed model is shown in Fig. 1. Blood entering the coronary circulation reaches the

From the Department of Surgery, St. Louis University School of Medicine, St. Louis, Mo.

Received for publication Feb. 25, 1993.

Accepted for publication May 4, 1993.

Address for reprints: D. Glenn Pennington, MD, St. Louis University Hospital, 3635 Vista Ave. at Grand Blvd., P.O. Box 15250, St. Louis, MO 63110-0250.

Copyright $\odot 1994$ by Mosby-Year Book, Inc.

$0022-5223 / 94 \$ 1.00+.10 \quad 12 / 1 / 49313$ coronary sinus and then the right ventricle, after which it passes through the right lung and enters the left atrium, left ventricle, and then the donor ascending aorta. Blood entering the left lung from the recipient aorta reaches the left atrium and mixes with the blood returned from the right lung. The donor left ventricle ejects into the recipient aorta. Therefore, the color of the donor left lung is bright red because of oxygenated arterial blood flow, and the two lobes of the transplanted right lung are dark red with desaturated coronary sinus blood.

Male inbred Lewis rats weighing 270 to $320 \mathrm{gm}$ were used as recipient and donor and the operation was performed in a sterile manner. The basic techniques were the same as those previously described for heterotopic heart and heart-lung transplantation. ${ }^{8-10}$ All animals received humane care in compliance with the "Guide for the Care and Use of Laboratory Animals" prepared by the Institute of Laboratory Animal Resources and published by the National Institutes of Health (NIH Publication No. 85-23, Revised 1985).

The donor rat was anesthetized with an intraperitoneal administration of sodium pentobarbital $(30 \mathrm{mg} / \mathrm{kg})$. Heparin $(300 \mathrm{U} / \mathrm{kg})$ was administered intravenously. The donor was placed in a supine position, and the chest and upper abdomen were prepared in the usual manner. A small midline abdominal incision was made, followed by a median sternotomy. A 14-gauge cannula was introduced into the right atrium through the suprahepatic inferior vena cava for infusion of cardioplegic solution. For these experiments we used $10 \mathrm{ml}$ of cold saline solution $\left(4^{\circ} \mathrm{C}\right)$ with potassium $20 \mathrm{mEq} / \mathrm{L}$ to arrest the heart. At the same time, cold saline solution was applied to the heart for topical cooling. After ligation and division of both superior venae cavae (including the azygos vein) with 5-0 silk sutures, the aorta was transected just distal to the origin of brachiocephalic artery. The heart and lungs were again flushed with $5 \mathrm{ml}$ of cold saline solution via the previously placed inferior vena cava cannula, and then the inferior vena cava was ligated and divided. 


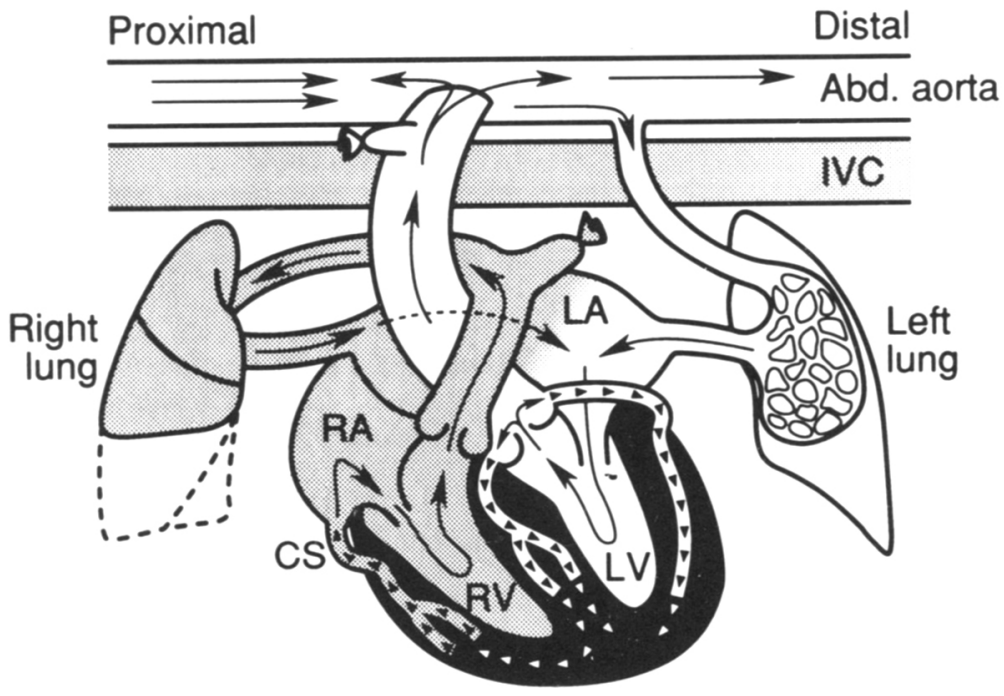

Fig. 1. Schema of the model: heterotopic, abdominally positioned heart-lung graft with donor ascending aorta and left pulmonary artery anastomosed to recipient abdominal aorta. Abd. aorta, Recipient's abdominal aorta; IVC, inferior vena cava; $L A$, left atrium; $L V$, left ventricle; $R A$, right atrium; $R V$, right ventricle; $C S$, coronary sinus.

Table I. Hemodynamics and left ventricular weight measurements

\begin{tabular}{|c|c|c|c|c|c|c|c|c|c|}
\hline \multirow[b]{2}{*}{ Model } & \multicolumn{2}{|c|}{$\begin{array}{c}H R \\
\text { (beats/min) }\end{array}$} & \multirow{2}{*}{$\begin{array}{c}L V S P \\
(m m H g) \\
T X \\
\end{array}$} & \multirow{2}{*}{$\begin{array}{c}E D P \\
(m m H g) \\
T X \\
\end{array}$} & \multirow{2}{*}{$\begin{array}{c}S V \\
(\mu l) \\
T X \\
\end{array}$} & \multicolumn{3}{|c|}{$\begin{array}{l}L V W \\
(m g)\end{array}$} & \multirow{2}{*}{$\begin{array}{c}m A o P \\
(m m H g) \\
I S\end{array}$} \\
\hline & $T X$ & $I S$ & & & & $\overline{T X}$ & $I S$ & $T X / I S$ & \\
\hline Heart* & 286 & 352 & 116 & 8.5 & 6 & 252 & 711 & 0.354 & 128 \\
\hline Heart-Lung $\dagger$ & 246 & 335 & 132 & 8.0 & 17 & 254 & 635 & 0.399 & 139 \\
\hline Working & 241 & 349 & 128 & 15.8 & 49 & 360 & 612 & 0.589 & 115 \\
\hline Working & 229 & 340 & 110 & 9.6 & 83 & 383 & 622 & 0.616 & 107 \\
\hline Working & 289 & 345 & 140 & 13.8 & 110 & 400 & 613 & 0.651 & 108 \\
\hline Working & 240 & 361 & 136 & 10.6 & 123 & 502 & 746 & 0.672 & 112 \\
\hline Working & 276 & 370 & 153 & 25.0 & 162 & 561 & 593 & 0.945 & 110 \\
\hline Working & 287 & 349 & 118 & 18.0 & 174 & 606 & 571 & 1.062 & 101 \\
\hline Working & 261 & 340 & 120 & 18.3 & 252 & 660 & 715 & 0.924 & 110 \\
\hline Working & 201 & 368 & 133 & 29.0 & 309 & 735 & 717 & 1.025 & 114 \\
\hline Working & 247 & 349 & 151 & 18.4 & 325 & 751 & 529 & 1.420 & 106 \\
\hline
\end{tabular}

$\overline{H R}$, Heart rate; $L V S P$, left ventricular systolic pressure; $E D P$, left ventricular end-diastolic pressure; $S V$, stroke volume; $L V W$, left ventricular weight; $m A O P$, mean aortic pressure; $T X$, transplanted heart; $I S$, in situ heart; $T X / I S$, left ventricular weight ratio.

${ }^{*}$ Heterotopic heart transplantation model $(n=1){ }^{8}$

${ }^{* *}$ Heterotopic heart-lung transplantation model $(n=1){ }^{9}$

The right lower and postcaval lobes (third and fourth lobes) were ligated at the hilum and resected. The main bronchi were divided and left open to allow drainage of secretions. The heartlung block was then freed by peeling off the esophagus. It was then immediately immersed in cold saline solution and transplanted into the recipient.

Anesthesia was initiated in the recipient in the same way as in the donor; however, no heparin was administered. The recipient was placed in the same position, and a midline abdominal incision was used to expose the infrarenal aorta. The aorta was crossclamped with a microvessel clip for the proximal site and a small bulldog clamp which was placed on both the aorta and vena cava as a unit for the distal site. Two longitudinal incisions were made in the aorta that were approximately equal to the diameter of the corresponding vessels to be anastomosed. Any clots found in the incised aorta were carefully removed.

The donor heart was placed in the left side of the abdominal cavity of the recipient. During the transplant procedure, the graft was wrapped in cold, wet gauze with an occasional application of cold saline solution. First, the donor aorta was anastomosed to the proximal incision in the recipient aorta in an end-to-side fashion with running 9-0 polypropylene suture. Then the donor left pulmonary artery was ligated and transected at its takeoff from the main pulmonary artery. The distal portion of the left pulmonary artery was anastomosed to the second smaller aortotomy similarly with 9-0 polypropylene 

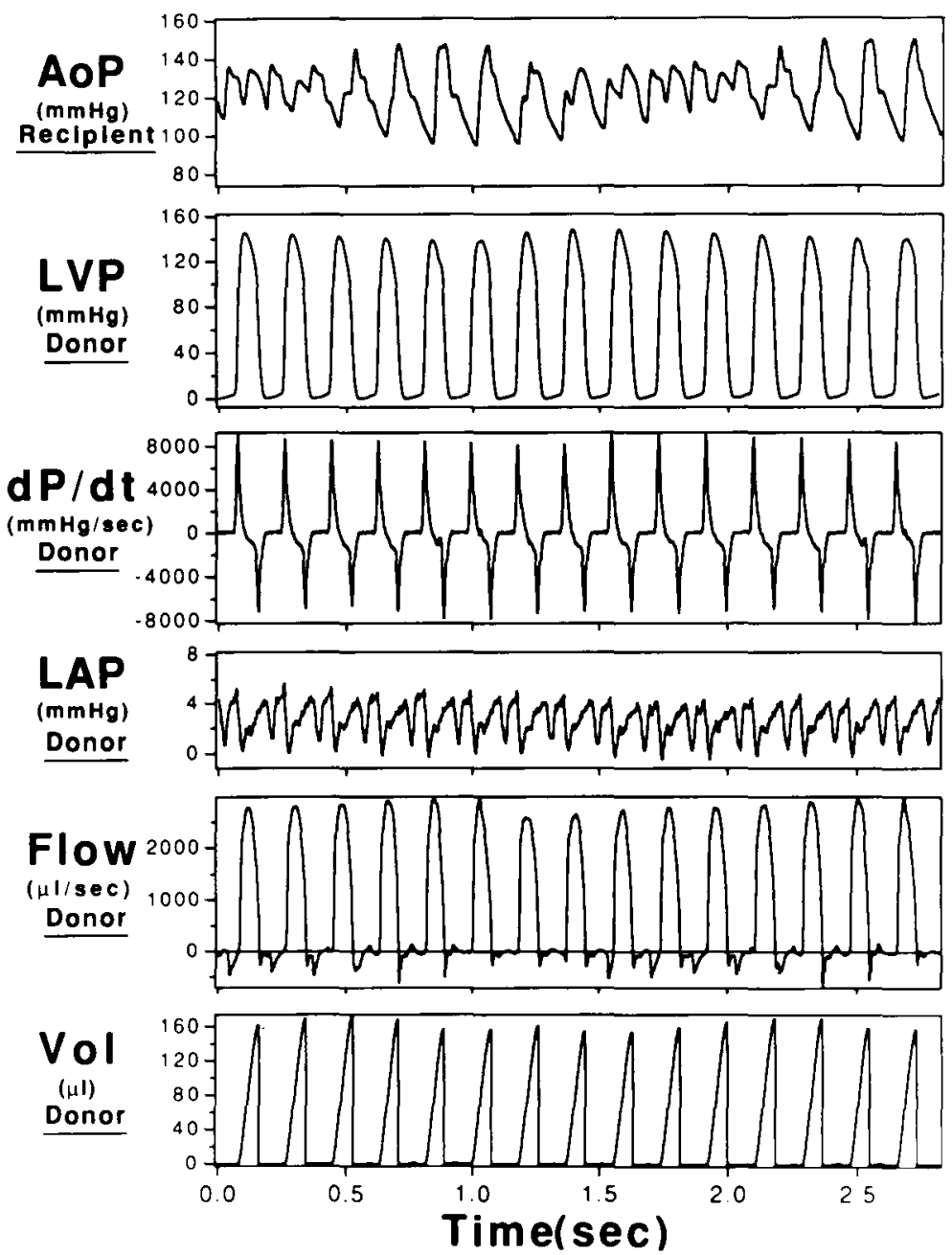

Fig. 2. Representative tracing of hemodynamic recordings. Aortic pressure tracing $(A O P)$ shows ejection from both donor and recipient left ventricles. $L V P$, Donor left ventricular pressure; $d P / d t$, first derivative of the donor left ventricular pressure; $L A P$, donor left atrial pressure; Flow, donor ascending aortic flow; $V o l$, ejected volume by the donor left ventricle (integration of the ejected flow).

suture. In these experiments, we intentionally varied anastomotic size to introduce different amounts of blood flow into the graft.

After completion of the anastomoses, a vascular clamp was placed on the left pulmonary artery to keep this route closed while air was evacuated from the donor ascending aorta by releasing the distal clamp. Then the proximal clamp on the recipient aorta was released, allowing reperfusion of the graft. The heart usually resumed beating spontaneously within 1 minute. After 15 to 20 minutes of reperfusion in an unloaded condition, the clamp on the left pulmonary artery was slowly released to introduce blood into the graft. If the heart was unable to handle the load, an additional 10 minutes of unloaded reperfusion was allowed. Usually, 30 minutes was long enough for the heart to recover even if there was a large amount of flow.

To prevent rotation or torsion of the graft within the abdomen, the graft was fixed to the retroperitoneal tissue with the ligature used for the right lobar resection. A small polytetrafluoroethylene patch* was placed around the donor ascending aorta to facilitate dissection and for placement of a flow probe used in future measurements. No antibiotics were administered. Graft function was monitored by palpation and surface echocardiogram.

Measurements. Hemodynamic measurements were made 4 to 5 weeks after transplantation. Anesthesia was induced and maintained by inhalation of isoflurane (Forane; Anaquest, Inc., Madison, Wis.) by mask. To minimize hemodynamic changes associated with anesthesia and instrumentation, we used minimal concentrations $(0.8 \%$ to $1 \%)$ of isoflurane. In addition, we

*Gore-Tex graft, is registered trademark of W. L. Gore \& Associates, Inc., Newark, Del. 
used diluted subcutaneous bupivacaine (Marcaine, Winthrop Laboratories Inc., New York, N.Y.) for the skin incision. Body temperature was kept constant by a warming plate.

Before laparotomy, a 22-gauge cannula was introduced into the left carotid artery, and its tip was positioned in the descending thoracic aorta. This aortic pressure was used as the reference for subsequent measurements.

The donor left ventricular pressure was measured with a $3 \mathrm{~F}$ high-fidelity catheter tip microtransducer (Millar Instruments, Houston, Tex.), which was inserted into the left ventricular cavity through the apex. Ejecting flow from the donor heart was measured with an ultrasonic transit-time flow probe (Transonic Systems, Ithaca, N.Y.) placed around the donor's ascending aorta. Blood pressure was adjusted to the prelaparotomy level by infusion of blood or saline solution. All signals were digitized and recorded on hard disk for off-line analysis. Stroke volumes were calculated by integrating the forward flow signals and were averaged every 10 cycles.

At the completion of these measurements, the animal was killed and the transplanted heart was removed. The left ventricle, including the intraventricular septum, was isolated and weighed in a blot-dried state after removal of the atrial tissue, great vessels, and right ventricular free wall.

\section{Results}

Nine operations were performed with this model. For comparison, conventional heart and heart-lung transplantation models were also created.

The average operating time was $78 \pm 5$ minutes, and the ischemic time was $35 \pm 4$ minutes. The average time of harvesting was 8 minutes. All rats survived at least 28 days after transplantation and were in good condition at the time they were killed. Hemodynamics and left ventricular weights before the animals were killed are shown in Table I. A representative tracing of the recipient aortic pressure, the donor left ventricular pressure, left ventricular rate of pressure rise, left atrial pressure, and donor ascending aortic flow with the integration of forward flow are shown in Fig. 2. The heart rates of the transplanted hearts were always lower than those of the recipients' hearts.

In this model, the left ventricular stroke volumes of the transplanted hearts ranged from 49 to $325 \mu \mathrm{l}$ and varied according to anastomotic size. Left ventricular weight of the transplanted heart showed a variable degree of atrophy, corresponding to stroke volume, and a significant correlation was found between stroke volume and left ventricular weight $(p<0.001$; Fig. 3) Left ventricular end-diastolic pressure was high in several animals, but, in the rest of the cases, it was in normal range. It is noteworthy that, even in the conventional heterotopic heart transplant model, the left ventricle ejected a small amount of blood every three to four beats. We observed viscous mucus in the bronchial tree of the graft; however, we did not observe any infections in this isograft series.

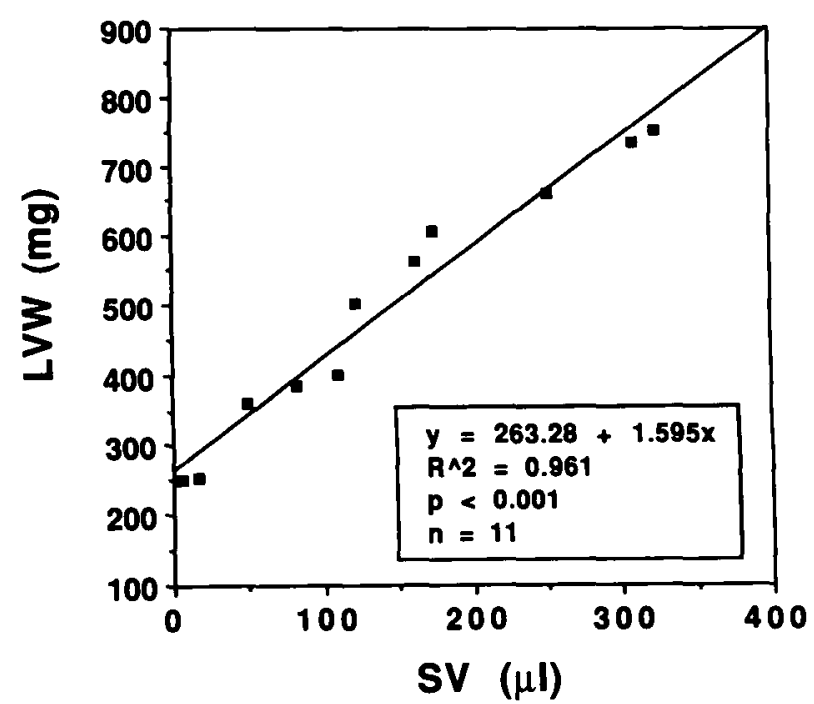

Fig. 3. Correlation between stroke volume and left ventricular weight. $S V$, Stroke volume; $L V W$, left ventricular weight.

\section{Discussion}

Some of the advantages of heterotopic rat heart transplantation are its close similarity to clinical conditions and its economy. The graft is perfused with blood and remains in a physiologic environment. ${ }^{6,7}$ Chronic management is much easier than that in large animals with considerably less cost. The life cycle is also much faster. Despite these advantages, ventricular unloading in previous models has limited value in many experimental studies.

Recently, attempts to load the transplanted heart have been reported by several investigators. Konertz, Semik, and Bernhard ${ }^{11}$ developed an intrathoracic working rat model in which the donor ascending aorta was anastomosed to the recipients descending aorta and the donor left atrium and main pulmonary artery were anastomosed to the recipients left atrium with a Dacron ring. Consistent survival with the use of this technique has not been subsequently reported.

Ding and associates ${ }^{12}$ anastomosed the donor left atrium to the distal recipient inferior vena cava in rabbits, and Ohmi and associates ${ }^{13}$ created a large intraatrial communication in the donor heart in dogs. Both of these methods introduced venous blood into the left heart as preload, which resulted in decreasing oxygen saturation in the donor ascending aorta. We cannot accurately judge the amount of loading or the adequacy of the oxygen supply-demand balance of the donor ventricle from these reports because the authors did not include stroke volume or flow from the donor left ventricle. Increasing donor left 
ventricular preload in these models decreases oxygen saturation while increasing oxygen demand of the left ventricle.

Another method of loading the left ventricle is by placement of a latex balloon in the left ventricle. ${ }^{14,15}$ The advantage of this method is that end-systolic pressurevolume relationships can be easily constructed. However, the balloon method includes isovolumic, nonphysiologic loading, and its accuracy in small animals has not been proven. ${ }^{16,17}$ Furthermore, management of the thebesian flow is difficult, especially in long-term series with the resultant prevalence of extensive thrombosis of the left ventricular cavity and endocardial fibrosis. ${ }^{14}$

The advantages of our working model are as follows: (1) The left ventricle ejects oxygenated arterial blood, which perfuses its own coronary circulation, and therefore it is not associated with decreasing oxygen saturation while increasing preload. (2) Because the loading blood returns to the recipient's abdominal aorta, the recipient does not receive any extra load; rather, the recipient heart may be unloaded because the graft works like an intraaortic pressure source, which is reflected in the low mortality of this model. This advantage also permits animals of disparate size to be used as donors and recipients. (3) The amount of preload is easily controlled by anastomosis size or by placing an external snare around the left pulmonary artery. This model can produce a wide range of stroke volumes, including normal values (Table I).

The success of this model is based on hemodynamic decoupling of the donor left atrium from the recipient arterial system by imposing the pulmonary vasculature. This decoupling provided not only adequate resistance for a pressure drop but also functioned as a reservoir. We initially connected the recipient's abdominal aorta to the donor left atrium with the use of a rat aortic conduit that was simultaneously harvested from the donor at the time of donor heart preparation. This worked well in shortterm experiments, but, in the long-term experiments, we observed high left atrial pressures with large blood flows or obstruction of the conduit. Donor left ventricle preload was unstable and greatly affected by a slight pressure difference between the recipient abdominal aorta and donor left atrium. This experience suggested the need for a hydrodynamic component between the donor left atrium and the recipient aorta. Interposing lung tissue solved this problem.

With respect to the hemodynamics of this model, high left ventricular end-diastolic pressure, which was observed in several cases, is alarming. This may be caused by inadequate myocardial preservation even if the ischemic time is short. However, our recent observation that increasing the donor heart rate decreases the end-diastolic pressure suggests that the inability of the donor heart to increase the intrinsic heart rate in relation to the load (as a result of denervation) $)^{4,6}$ is also involved.

The limitation of this model is the presence of lung tissue. For certain studies, such as those related to long-term preservation or rejection, this tissue may affect the experimental results. We are currently evaluating another long-term loading method in which aortic regurgitation is created in the transplanted heart by destroying one aortic cusp. This may circumvent the lung problem.

In summary, we developed a working transplanted heart model in which the transplanted left ventricle ejects oxygenated blood over a wide range of stroke volumes. This economic model may allow long-term assessments of cardiac remodeling, long-term efficacy of cardiac preservation, and posttransplantation ventricular performance.

\section{REFERENCES}

1. Adams DH, Tilney NL, Collins JJ Jr, Karnovsky MJ. Experimental graft arteriosclerosis: the Lewis-to-F-344 allograft model. Transplantation 1992;53:1115-9.

2. McGregor CG, McCallum HM, Hannan J, et al. Longterm effects of cold cardioplegic myocardial protection in the rat. J Thorac Cardiovasc Surg 1984;87:913-9.

3. Klein I, Hong C, Schreiber SS. Isovolumic loading prevents atrophy of the heterotopically transplanted rat heart. Circ Res 1991;69:1421-5.

4. Geenen DL, Malhotra A, Buttrick PM, Scheuer J. Increased heart rate prevents the isomyosin shift after cardiac transplantation in the rat. Circ Res 1992;70:554-8.

5. Korecky B, Masika M. Direct effect of increased hemodynamic load on cardiac mass. Circ Res 1991;68:1174-8.

6. Klein I, Hong C, Schreiber SS. Cardiac atrophy in the heterotopically transplanted rat heart: in vitro protein synthesis. J Mol Cell Cardiol 1990;22:461-8.

7. Korecky B, Zak R, Schwartz K, Aschenbrenner V. Role of thyroid hormone in regulation of isomyosin composition, contractility, and size of heterotopically isotransplanted rat heart. Circ Res 1987;60:8824-30.

8. Ono K, Lindsey ES. Improved technique of heart transplantation in rats. J THORAC Cardiovasc Surg 1969; 57:225-9.

9. Fox U, Montorsi M. A technical modification of heart-lung transplantation in rats. J Microsurg 1980;1:377-80.

10. Lee S, Macedo AR, Curtis GP, et al. A simplified model for heterotopic rat heart transplantation. Transplantation 1982;33:438-42.

11. Konertz W, Semik M, Bernhard A. Heart, lung and heartlung transplantation in rats. J Heart Transplant 1985; 4:426-30.

12. Ding $\mathrm{M}$, Dyke CM, Kadletz $\mathrm{M}$, et al. A working heterotopic heart transplantation model. Surg Forum 1992;43: 241-3. 
13. Ohmi M, Yokoyama H, Nakame T, et al. Hemodynamic performance in a heterotopically transplanted dog heart: proposal of techniques for working left heart model of heterotopic (abdominal) heart transplantation. J Heart Lung Transplant 1992;11:1147-50.

14. Galinanes M, Hearse DJ. Metabolic, functional, and histologic characterization of the heterotopically transplanted rat heart when used as a model for the study of long-term recovery from global ischemia. $\mathbf{J}$ Heart Lung Transplant 1991;10:79-91.

15. Sun SC, Appleyard R, Masetti P, et al. Improved recovery of heart transplants by combined use of oxygen-derived free radical scavengers and energy enhancement. J THORAC CARDIOVASC SuRg 1992;104:830-37.

16. Goto Y, Slinker BK, LeWinter MM. Accuracy of volume measurement of rabbit left ventricle by balloon method. Am J Physiol 1988;255:H394-6.

17. Wannenburg T, Schulman SP, Burkhoff D. End-systolic pressure-volume and MVO2-pressure-volume area relations of isolated rat hearts. Am J Physiol 1992;262:H128793.

\section{Bound volumes available to subscribers}

Bound volumes of THE JOURNAL OF THORACIC AND CARDIOvaSCULAR SURGERY are available to subscribers (only) for the 1994 issues from the Publisher, at a cost of $\$ 82.50$ for domestic, $\$ 106.28$ for Canadian, and $\$ 100.50$ for international subscribers for Vol. 107 (January-June) and Vol. 108 (July-December). Shipping charges are included. Each bound volume contains a subject and author index and all advertising is removed. Copies are shipped within 60 days after publication of the last issue of the volume. The binding is durable buckram with the JOURNAL name, volume number, and year stamped in gold on the spine. Payment must accompany all orders. Contact Mosby-Year Book, Inc., Subscription Services, 11830 Westline Industrial Drive, St. Louis, Missouri 63146-3318, USA; phone 1 (800) 4534351 or (314) $453-4351$.

Subscriptions must be in force to qualify. Bound volumes are not available in place of a regular JourNAL subscription. 